\title{
Analytical investigation of the phase transition between holographic insulator and superconductor in Gauss-Bonnet gravity
}

\author{
Qiyuan Pan $^{1,2 *}$, Jiliang Jing ${ }^{1,2 \dagger}$, Bin Wang ${ }^{3 \ddagger}$ \\ ${ }^{1}$ Institute of Physics and Department of Physics, \\ Hunan Normal University, Changsha, Hunan 410081, China \\ ${ }^{2}$ Key Laboratory of Low Dimensional Quantum Structures and Quantum Control of Ministry of Education, \\ Hunan Normal University, Changsha, Hunan 410081, China and \\ ${ }^{3}$ INPAC and Department of Physics, Shanghai Jiao Tong University, Shanghai 200240, China
}

\begin{abstract}
We employ the variational method for the Sturm-Liouville eigenvalue problem to analytically study the phase transition between the holographic insulator and superconductor in the GaussBonnet gravity. By investigating the s-wave and p-wave holographic insulator/superconductor models, we find that this analytic method is more effective to obtain the analytic results on the condensation and the critical phenomena in the AdS soliton background in Gauss-Bonnet gravity. Our analytic result can be used to back up the numerical computations in the AdS soliton with Gauss-Bonnet correction.
\end{abstract}

PACS numbers: $11.25 . \mathrm{Tq}, 04.70 . \mathrm{Bw}, 74.20 .-\mathrm{z}$

* panqiyuan@126.com

† jljing@hunnu.edu.cn

¥ wang b@sjtu.edu.cn 


\section{INTRODUCTION}

The profound finding of the anti-de Sitter/conformal field theory (AdS/CFT) correspondence [1 3] has provided a framework to describe the strongly coupled field theories in a weakly coupled gravitational system. Recently, this correspondence has been applied to study the holographic model of superconductors in which a remarkable connection has been observed between the gravitational physics and the condensed matter physics 4]. It has been shown that the bulk AdS black hole becomes unstable and scalar hair condenses below a critical temperature. The instability of the bulk black hole corresponds to a second order phase transition from normal state to superconducting state which brings the spontaneous $\mathrm{U}(1)$ symmetry breaking. In the boundary dual CFT, these properties exhibit the behavior of the superconductor [5, 6]. Due to the potential applications to the condensed matter physics, the condensation in bulk AdS black holes has been investigated extensively, for reviews, see Refs. [7-9] and references therein.

In additional to the bulk AdS black hole spacetime, recently it was found that a holographic model can be constructed in the bulk AdS soliton background to describe the insulator and superconductor phase transition [10]. Adding the chemical potential to the AdS soliton, a second order phase transition can happen when the chemical potential is over a critical value. This phase transition can be used to describe the transition between the insulator and superconductor, while it is different from the Hawking-Page phase transition between the Ricci flat AdS black hole and the AdS soliton [11]. Taking the backreaction of the matter fields into account, it was argued that the order of the phase transition can be changed from the second to the first if the backreaction is strong enough [12]. In the Stückelberg mechanism, rich physics on the phase transition between the holographic insulator and superconductor in the AdS soliton background has been observed [13]. Further investigations on various insulator and superconductor phase transitions in different theories of gravity have been carried out [14 18$]$.

In most cases, the holographic superconductors were studied numerically. Ideally, one would like to have a full analytic description of the phase transition and condensation phenomena. In addition to back up numerical results, the analytic description can help to gain more insights, for example it may tell what properties of the action decide the mean-field behaviors etc. Recently, there appeared two analytic approaches in parallel to the numerical calculation. One is the analytic matching method which was first proposed in [19] and later refined

in [18, 20]. With this method, we can calculate the critical temperature analytically within a few percent in 
the best case. This analytic approach has been extended to derive the upper critical magnetic field when the holographic superconductor is immersed in constant external magnetic fields [21, 22]. In higher dimensions, this analytic method can keep valid only when the matching point is chosen within an appropriate range [18]. However the matching method is not effective to describe the AdS soliton, neither can it be used to derive the critical exponents for the condensation near the critical temperature. The mean-field critical exponent $1 / 2$ at the critical temperature comes mostly from numerically solving the holographic systems and doing data fitting. In [23, 24], the authors extended the variational method for the Sturm-Liouville (S-L) eigenvalue problem to analytically calculate the critical exponent near the critical temperature. This method was further applied to analytically study some properties of holographic superconductors in AdS black hole backgrounds in Einstein gravity [25] and Einstein-Gauss-Bonnet gravity [26] in the probe limit, respectively.

An analytic study by using the S-L method on the phase transition between the holographic insulator and superconductor was done in [17] in the Einstein gravity. It is of interest to further generalize the S-L method to study holographic superconductor developed in the AdS soliton background in the Gauss-Bonnet gravity. The condensation phenomena and the phase transition between the s-wave holographic insulator and superconductor in the Gauss-Bonnet gravity were investigated numerically in [18, 27]. We will also extend the investigation of the p-wave holographic insulator and superconductor phase transition with Gauss-Bonnet correction in this work, which has not been constructed as far as we know. It is not trivial to analytically study the condensation and the phase transition by taking into account of the influence of the Gauss-Bonnet coupling. Besides to be used to check numerical computation, the analytic investigation can clearly disclose the critical exponent of the system at the critical temperature and the influence of the Gauss-Bonnet factor in the phase transition. In the AdS soliton background, we will compare two available analytic methods and argue that the S-L method is more effective for the analytic study of the condensation.

The plan of the work is the following. In Sec. II we briefly review the AdS soliton background in the Gauss-Bonnet gravity. In Sec. III we explore the s-wave insulator and superconductor phase transition with Gauss-Bonnet correction. In Sec. IV we discuss the p-wave case. We conclude in the last section with our main results. 


\section{GAUSS-BONNET ADS SOLITON}

In order to study the superconducting phase dual to the Guass-Bonnet AdS soliton configuration in the probe limit, we start with the five-dimensional AdS soliton in the Gauss-Bonnet gravity in the form [28]

$$
d s^{2}=-r^{2} d t^{2}+\frac{d r^{2}}{f(r)}+f(r) d \varphi^{2}+r^{2}\left(d x^{2}+d y^{2}\right)
$$

with

$$
f(r)=\frac{r^{2}}{2 \alpha}\left[1-\sqrt{1-\frac{4 \alpha}{L^{2}}\left(1-\frac{r_{s}^{4}}{r^{4}}\right)}\right],
$$

where $r_{s}$ is the tip of the soliton which is a conical singularity in this solution, $\alpha$ is the Gauss-Bonnet coupling constant and $L$ is the AdS radius. It should be noted that in the asymptotic region $(r \rightarrow \infty)$, we find

$$
f(r) \sim \frac{r^{2}}{2 \alpha}\left(1-\sqrt{1-\frac{4 \alpha}{L^{2}}}\right),
$$

so the effective asymptotic AdS scale can be defined by 28, 29]

$$
L_{\mathrm{eff}}^{2}=\frac{2 \alpha}{1-\sqrt{1-\frac{4 \alpha}{L^{2}}}} \rightarrow \begin{cases}L^{2}, & \text { for } \alpha \rightarrow 0, \\ \frac{L^{2}}{2}, & \text { for } \alpha \rightarrow \frac{L^{2}}{4}\end{cases}
$$

$\alpha=L^{2} / 4$ is the Chern-Simons limit corresponding to the upper bound of the Gauss-Bonnet factor. When

$\alpha \rightarrow 0$, (11) goes back to the Schwarzschild AdS soliton. For simplicity, in the following we will consider the Gauss-Bonnet factor in the range $0<\alpha \leq L^{2} / 5$. For the smoothness at the tip, we impose a period $\beta=\frac{4 \pi L^{2}}{(d-1) r_{s}}$ for the coordinate $\varphi$ to remove the singularity.

\section{PHASE TRANSITION BETWEEN THE S-WAVE INSULATOR AND SUPERCONDUCTOR}

In the background of the Gauss-Bonnet-AdS soliton, we consider a Maxwell field and a charged complex scalar field coupled via the action

$$
S=\int d^{5} x \sqrt{-g}\left[-\frac{1}{4} F_{\mu \nu} F^{\mu \nu}-|\nabla \psi-i A \psi|^{2}-m^{2}|\psi|^{2}\right]
$$

Taking the ansatz of the matter fields as $\psi=\psi(r)$ and $A=\phi(r) d t$, we can get the equations of motion for the scalar field $\psi$ and gauge field $\phi$ in the form

$$
\begin{gathered}
\psi^{\prime \prime}+\left(\frac{f^{\prime}}{f}+\frac{3}{r}\right) \psi^{\prime}+\left(\frac{\phi^{2}}{r^{2} f}-\frac{m^{2}}{f}\right) \psi=0 \\
\phi^{\prime \prime}+\left(\frac{f^{\prime}}{f}+\frac{1}{r}\right) \phi^{\prime}-\frac{2 \psi^{2}}{f} \phi=0
\end{gathered}
$$


where the prime denotes the derivative with respect to $r$.

In order to solve the above equations, we have to impose the boundary conditions at the tip $r=r_{s}$ and at $r \rightarrow \infty$. At the tip $r=r_{s}$, the solutions behave as

$$
\begin{gathered}
\psi=\tilde{\psi}_{0}+\tilde{\psi}_{1}\left(r-r_{s}\right)+\tilde{\psi}_{2}\left(r-r_{s}\right)^{2}+\cdots, \\
\phi=\tilde{\phi}_{0}+\tilde{\phi}_{1}\left(r-r_{s}\right)+\tilde{\phi}_{2}\left(r-r_{s}\right)^{2}+\cdots,
\end{gathered}
$$

where $\tilde{\psi}_{i}$ and $\tilde{\phi}_{i}(i=0,1,2, \cdots)$ are integration constants, and the Neumann-like boundary condition has been imposed to keep every physical quantity finite [10]. It is worth noticing that one can find a constant nonzero gauge field $\phi\left(r_{s}\right)$ at $r=r_{s}$, in contrary to that of the AdS black hole where $\phi\left(r_{+}\right)=0$ at the horizon.

Near the boundary $r \rightarrow \infty$, we have asymptotic behaviors

$$
\psi=\frac{\psi_{-}}{r^{\lambda_{-}}}+\frac{\psi_{+}}{r^{\lambda_{+}}}, \quad \phi=\mu-\frac{\rho}{r^{2}}
$$

where $\mu$ and $\rho$ are interpreted as the chemical potential and charge density in the dual field theory respectively.

Here $\lambda_{ \pm}=2 \pm \sqrt{2+m^{2} L_{\text {eff }}^{2}}$. The coefficients $\psi_{-}$and $\psi_{+}$both multiply normalizable modes of the scalar field equations and they correspond to the vacuum expectation values $\psi_{-}=<\mathcal{O}_{-}>, \psi_{+}=<\mathcal{O}_{+}>$of operators dual to the scalar field according to the AdS/CFT correspondence. We can impose boundary conditions that either $\psi_{-}$or $\psi_{+}$vanish [5, 6]. For simplicity, we will scale $L=1$ and $r_{s}=1$ in the following just as in [10, 18].

Before going further, we would like to give a comment. In the AdS soliton background, since at the tip $\phi\left(r_{s}\right)$ does not vanish in (6) and (7), which leads that the $\phi^{2}$ terms in the coupled equations cannot be got rid of as did in the AdS black hole case, we cannot count on the matching method to obtain the analytic result. Here we will apply the S-L method [23] to analytically investigate the properties of the s-wave holographic insulator/superconductor phase transition in the Gauss-Bonnet gravity. We will calculate the critical chemical potential to accommodate the phase transition and analytically derive the critical exponent of condensation operator. In addition, we will derive the relation between the charge density and the chemical potential near the phase transition point and examine the effect of the Gauss-Bonnet factor.

\section{A. Critical chemical potential}

Introducing a new variable $z=r_{s} / r$, we can rewrite Eqs. (6) and (7) into

$$
\begin{gathered}
\psi^{\prime \prime}+\left(\frac{f^{\prime}}{f}-\frac{1}{z}\right) \psi^{\prime}+\left(\frac{\phi^{2}}{z^{2} f}-\frac{m^{2}}{z^{4} f}\right) \psi=0, \\
\phi^{\prime \prime}+\left(\frac{f^{\prime}}{f}+\frac{1}{z}\right) \phi^{\prime}-\frac{2 \psi^{2}}{z^{4} f} \phi=0
\end{gathered}
$$


where the prime here denotes the derivative with respective to $z$.

It has been shown numerically that the solution is unstable and a hair can be developed when the chemical potential is bigger than a critical value, i.e., $\mu>\mu_{c}$. For lower chemical potential, $\mu<\mu_{c}$, the gravitational dual is an AdS soliton with a nonvanishing profile for the scalar field $\psi$, which can be viewed as an insulator phase [10, 18]. Thus, there is a phase transition between the insulator and superconductor phases around the critical chemical potential $\mu_{c}$.

At the critical chemical potential $\mu_{c}$, the scalar field $\psi=0$. So near the critical point Eq. (11) reduces to

$$
\phi^{\prime \prime}+\left(\frac{f^{\prime}}{f}+\frac{1}{z}\right) \phi^{\prime}=0
$$

With the Neumann-like boundary condition (8) for the gauge field $\phi$ at the tip $r=r_{s}$, we can obtain the physical solution $\phi(z)=\mu$ to Eq. (12) when $\mu<\mu_{c}$. Considering the asymptotic behavior in Eq. (9), close to the critical point this solution indicates that $\rho=0$ near the AdS boundary $z=0$, which gives fairly good agreement with numerical results in Ref. [18].

As $\mu \rightarrow \mu_{c}$, the scalar field equation (10) reduces to

$$
\psi^{\prime \prime}+\left(\frac{f^{\prime}}{f}-\frac{1}{z}\right) \psi^{\prime}+\left(\frac{\mu^{2}}{z^{2} f}-\frac{m^{2}}{z^{4} f}\right) \psi=0
$$

As in [23], we introduce a trial function $F(z)$ near the boundary $z=0$ which satisfies

$$
\psi(z) \sim\left\langle\mathcal{O}_{i}\right\rangle z^{\lambda_{i}} F(z)
$$

with $i=+$ or $i=-$. Here we will impose the boundary condition $F(0)=1$ and $F^{\prime}(0)=0$. Then, we can obtain the equation of motion for $F(z)$

$$
F^{\prime \prime}+\left[\frac{2 \lambda_{i}}{z}+\left(\frac{f^{\prime}}{f}-\frac{1}{z}\right)\right] F^{\prime}+\left[\frac{\lambda_{i}\left(\lambda_{i}-1\right)}{z^{2}}+\frac{\lambda_{i}}{z}\left(\frac{f^{\prime}}{f}-\frac{1}{z}\right)+\frac{1}{z^{4} f}\left(\mu^{2} z^{2}-m^{2}\right)\right] F=0 .
$$

Defining a new function

$$
T(z)=\frac{z^{2 \lambda_{i}-3} \sqrt{1+4\left(z^{4}-1\right) \alpha}-1}{2 \sqrt{\alpha}}
$$

we can rewrite Eq. (15) as

$$
\left(T F^{\prime}\right)^{\prime}+T\left[\frac{\lambda_{i}\left(\lambda_{i}-1\right)}{z^{2}}+\frac{\lambda_{i}}{z}\left(\frac{f^{\prime}}{f}-\frac{1}{z}\right)+\frac{1}{z^{4} f}\left(\mu^{2} z^{2}-m^{2}\right)\right] F=0 .
$$

According to the Sturm-Liouville eigenvlaue problem [30], we obtain the expression which can be used to estimate the minimum eigenvalue of $\mu^{2}$

$$
\mu^{2}=\frac{\int_{0}^{1} T\left(F^{\prime 2}-U F^{2}\right) d z}{\int_{0}^{1} V F^{2} d z}
$$


with

$$
\begin{aligned}
U & =\frac{\lambda_{i}\left(\lambda_{i}-1\right)}{z^{2}}+\frac{\lambda_{i}}{z}\left(\frac{f^{\prime}}{f}-\frac{1}{z}\right)-\frac{m^{2}}{z^{4} f}, \\
V & =\frac{T}{z^{2} f} .
\end{aligned}
$$

In the following calculation, we will assume the trial function to be $F(z)=1-a z^{2}$, where $a$ is a constant.

In [18] the condensate $\left\langle\mathcal{O}_{+}\right\rangle$was numerically calculated in 5-dimensional Gauss-Bonnet AdS soliton background simply by fixing $\psi_{-}=0$. It has been shown numerically that the increase of the Gauss-Bonnet factor $\alpha$ results in the increase of the critical chemical potential, which means that the higher curvature correction will make it harder for the scalar hair to be condensated. Now we can use the S-L method to understand the condensation analytically.

Using Eq. (18) to compute the minimum eigenvalue of $\mu^{2}$ for $i=+$, we can obtain the critical chemical potential $\mu_{c}$ for different strength of the curvature correction and the mass of the scalar field. As an example, we calculate the case of $m^{2} L_{\text {eff }}^{2}=0$ in detail. From Eq. (18), we obtain

$$
\mu^{2}=\frac{\Sigma(a, \alpha)}{\Xi(a, \alpha)},
$$

with

$$
\begin{aligned}
& \left.\Sigma(a, \alpha)=\frac{1-6 \alpha+\sqrt{1-4 \alpha}(-1+4 \alpha)}{3 \alpha^{3 / 2}}+\frac{1}{16 \alpha^{2}}\left[2 \sqrt{\alpha}(-3+20 \alpha)+3(1-4 \alpha)^{2} \log \frac{\sqrt{\alpha}+2 \alpha}{\sqrt{\alpha(1-4 \alpha}}\right)\right] a \\
& +\frac{3\left[-1+10 \alpha-30 \alpha^{2}+\sqrt{1-4 \alpha}\left(1-8 \alpha+16 \alpha^{2}\right)\right]}{40 \alpha^{5 / 2}} a^{2}, \\
& \Xi(a, \alpha)=\frac{\left(-10+15 a-6 a^{2}\right) \sqrt{\alpha}}{60} \text {. }
\end{aligned}
$$

For different values of the Gauss-Bonnet factor, we can get the minimum eigenvalues of $\mu^{2}$ and the corresponding values of $a$, for example, $\mu_{\text {min }}^{2}=11.607$ and $a=0.440$ for $\alpha=0.0001, \mu_{m i n}^{2}=12.667$ and $a=0.386$ for $\alpha=0.1$ and $\mu_{\text {min }}^{2}=14.365$ and $a=0.257$ for $\alpha=0.2$. Then, we have the critical chemical potential $\mu_{c}=\mu_{\min }$ [17]. In Table【 we present the critical chemical potential $\mu_{c}$ for chosen values of the Gauss-Bonnet coupling $\alpha$ and various masses of the scalar field determined by fixing $m^{2} L_{\text {eff }}^{2}$. Comparing with numerical results, we find that the analytic results derived from S-L method are in good agreement with the numerical calculation.

From Table I, we observe that for the same mass of the scalar field, the critical chemical potential increases when the Gauss-Bonnet factor $\alpha$ becomes bigger. Our analytic result supports the observation obtained numerically that the higher curvature correction can make the scalar hair more difficult to be developed $18-$ 22, 27, 31 36]. It is interesting to note that the analytical S-L method can give consistent critical chemical 
TABLE I: The critical chemical potential $\mu_{c}$ obtained by the analytical S-L method (left column) and from numerical calculation (right column) with chosen Gauss-Bonnet coupling and various masses of the scalar field for the s-wave holographic insulator and superconductor model. In order to compare with the results in Refs. 10, 18], we also present the critical chemical potential for $m^{2} L_{\text {eff }}^{2}=-15 / 4$.

\begin{tabular}{ccccccc}
\hline$\alpha$ & \multicolumn{2}{c}{0.0001} & \multicolumn{2}{c}{0.1} & \multicolumn{2}{c}{0.2} \\
\hline$m^{2} L_{\text {eff }}^{2}=0$ & 3.407 & 3.404 & 3.559 & 3.556 & 3.790 & 3.789 \\
$m^{2} L_{\text {eff }}^{2}=-1$ & 3.137 & 3.135 & 3.275 & 3.272 & 3.477 & 3.475 \\
$m^{2} L_{\text {eff }}^{2}=-2$ & 2.817 & 2.815 & 2.937 & 2.935 & 3.106 & 3.105 \\
$m^{2} L_{\text {eff }}^{2}=-3$ & 2.399 & 2.396 & 2.497 & 2.494 & 2.624 & 2.622 \\
$m^{2} L_{\text {eff }}^{2}=-15 / 4$ & 1.897 & 1.888 & 1.963 & 1.960 & 2.042 & 2.039 \\
\hline
\end{tabular}

potential with the numerical result even when the mass of the scalar field is zero. This shows that the S-L method is more effective than the matching method. It was realized that the matching method cannot deal with the scalar field with zero mass [18], since for this case the Gauss-Bonnet term does not contribute to the analytic approximation in the matching method [18, 19].

For the same strength of the curvature correction, with the increase of the mass of scalar field, the critical chemical potential $\mu_{c}$ becomes larger. This property also agrees well with the numerical result [18].

In the AdS black hole in Gauss-Bonnet gravity [26], the S-L method was applied by choosing specific mass of the scalar field by fixing $m^{2} L_{e f f}^{2}=-3$. For choosing other nonzero values of the mass of scalar field, (4.16) there cannot be integrated analytically so that their (4.17), (4.18) and further steps cannot be derived. In the AdS soliton background, we found a much better situation. (18) above can be integrated analytically when the mass of the scalar field satisfying the Breitenlohner-Freedman bound [37], so that analytically we can observe the condensation with the change of the mass of the scalar field.

If we fix the scalar field mass by choosing values of $m^{2} L^{2}$ instead of $m^{2} L_{\text {eff }}^{2}$, the S-L method can give the same qualitative dependence of the critical chemical potential on the Gauss-Bonnet factor as described above when we study the scalar operator $\left\langle\mathcal{O}_{+}\right\rangle$. This supports the numerical computation in the AdS black hole background [18, 19].

Now we concentrate on the scalar operator $\left\langle\mathcal{O}_{-}\right\rangle$by imposing the condition $\psi_{+}=0$. Fixing the mass of the scalar field by choosing values of $m^{2} L_{\text {eff }}^{2}$, the scalar operator $\left\langle\mathcal{O}_{-}\right\rangle$presents us qualitatively the same behavior of the condensation when we change the strength of the curvature correction as we observed above. With the increase of the Gauss-Bonnet coupling, the critical chemical potential will increase, which shows that the condensation will be harder to develop. But if we fix the mass of the scalar field by choosing values of $m^{2} L^{2}$, analytically we observed completely different condensation behavior as the Gauss-Bonnet coupling changes, see Table II]. Our analytical result got by using S-L method presents the same abnormal behavior as found numerically in [18]. Considering that choosing the mass of the scalar field by selecting the value of $m^{2} L_{\text {eff }}^{2}$ 
contains directly the signature of Gauss-Bonnet factor in the scalar mass, we believe that this way of choosing the scalar field mass can disclose the correct consistent influence due to the Gauss-Bonnet coupling in various condensates.

TABLE II: The critical chemical potential $\mu_{c}$ obtained by using the analytical S-L method for the s-wave holographic insulator and superconductor model. The mass of scalar field is chosen by fixing $m^{2} L_{\text {eff }}^{2}=-15 / 4$ and $m^{2} L^{2}=-15 / 4$, respectively.

\begin{tabular}{crlll}
\hline$\alpha$ & 0.0001 & 0.01 & 0.05 & 0.1 \\
\hline$m^{2} L_{\text {eff }}^{2}=-15 / 4$ & 0.837 & 0.839 & 0.849 & 0.862 \\
\hline$m^{2} L^{2}=-15 / 4$ & 0.836 & 0.798 & 0.648 & 0.463 \\
\hline
\end{tabular}

\section{B. Critical phenomena}

We will use the S-L method to analytically discuss the critical phenomena for the phase transition between the s-wave holographic insulator and superconductor in the Gauss-Bonnet gravity. We will concentrate on studying the critical exponent for condensation operator and the relations between the charge density and the chemical potential.

The scalar field $\psi$ can be given by Eq. (14) when $\mu \rightarrow \mu_{c}$, so we can rewrite the equations of motion (11) as

$$
\phi^{\prime \prime}+\left(\frac{f^{\prime}}{f}+\frac{1}{z}\right) \phi^{\prime}-\frac{2\left\langle\mathcal{O}_{i}\right\rangle^{2} z^{2 \lambda_{i}-4} F^{2}}{f} \phi=0
$$

Since the condensation for the scalar operator $\left\langle\mathcal{O}_{i}\right\rangle$ is so small, we can expand $\phi(z)$ in small $\left\langle\mathcal{O}_{i}\right\rangle$ as

$$
\phi(z) \sim \mu_{c}+\left\langle\mathcal{O}_{i}\right\rangle \chi(z)+\cdots
$$

Considering the boundary condition at the tip, we can get $\chi(1)=0$ and $\chi^{\prime}(1)=$ constant. After defining a function

$$
P(z)=\frac{\sqrt{1+4\left(z^{4}-1\right) \alpha}-1}{2 \sqrt{\alpha} z}
$$

we can obtain the equation of motion for $\chi(z)$

$$
\left(P \chi^{\prime}\right)^{\prime}-2\left\langle\mathcal{O}_{i}\right\rangle \mu_{c} \frac{z^{2 \lambda_{i}-4} P F^{2}}{f}=0 .
$$

According to the asymptotic behavior in Eq. (9), we can expand $\phi$ when $z \rightarrow 0$ as

$$
\phi(z) \simeq \mu-\rho z^{2} \simeq \mu_{c}+\left\langle\mathcal{O}_{i}\right\rangle\left[\chi(0)+\chi^{\prime}(0) z+\frac{1}{2} \chi^{\prime \prime}(0) z^{2}+\cdots\right]
$$

From the coefficients of the $z^{0}$ term, we can easily get

$$
\mu-\mu_{c} \simeq\left\langle\mathcal{O}_{i}\right\rangle \chi(0)
$$


If we set

$$
\chi(z)=2\left\langle\mathcal{O}_{i}\right\rangle \mu_{c} \xi(z)
$$

where the function $\xi(z)$ is the solution to the following equation

$$
\xi^{\prime \prime}+\left(\frac{f^{\prime}}{f}+\frac{1}{z}\right) \xi^{\prime}-\frac{z^{2 \lambda_{i}-4} F^{2}}{f}=0,
$$

we will know that

$$
\left\langle\mathcal{O}_{i}\right\rangle=\frac{1}{\left[2 \mu_{c} \xi(0)\right]^{1 / 2}}\left(\mu-\mu_{c}\right)^{1 / 2}
$$

where $\xi(0)=c_{1}-\int_{0}^{1}\left[c_{2}+\int_{1}^{z} F(x)^{2} x^{2 \lambda_{i}-3} d x\right] \frac{d z}{z f(z)}$ with the integration constants $c_{1}$ and $c_{2}$ determined by the boundary condition $\chi(z)$. For example, fixing $m^{2} L_{\text {eff }}^{2}=-15 / 4$ and $\alpha=0.0001$, we can get $\xi(0)=0.0815$ when $a=0.330$ which results in $\left\langle\mathcal{O}_{+}\right\rangle \approx 1.801\left(\mu-\mu_{c}\right)^{1 / 2}$. This agrees well with the result given in [17].

Note that our expression (30) is valid for all cases considered here, thus near the critical point, both of the scalar operators $\left\langle\mathcal{O}_{+}\right\rangle$and $\left\langle\mathcal{O}_{-}\right\rangle$satisfy $\left\langle\mathcal{O}_{i}\right\rangle \sim\left(\mu-\mu_{c}\right)^{1 / 2}$. This behavior holds for various values of GaussBonnet couplings and masses of the scalar field. The analytic result supports the numerical computation [18, 27] that the phase transition between the s-wave holographic insulator and superconductor belongs to the second order and the critical exponent of the system takes the mean-field value $1 / 2$. The Gauss-Bonnet coupling will not influence the result.

Considering the coefficients of $z^{1}$ terms in Eq. (26), we find that $\chi^{\prime}(0) \rightarrow 0$ if $z \rightarrow 0$, which is consistent with the following relation by integrating both sides of Eq. (25)

$$
\left.\left[\frac{\chi^{\prime}(z)}{z}\right]\right|_{z \rightarrow 0}=-\frac{4 \sqrt{\alpha}\left\langle\mathcal{O}_{i}\right\rangle \mu_{c}}{\sqrt{1-4 \alpha}-1} \int_{0}^{1} \frac{z^{2 \lambda_{i}-4} P F^{2}}{f} d z
$$

Comparing the coefficients of the $z^{2}$ term in Eq. (26), we can express $\rho$ as

$$
\rho=-\frac{1}{2}\left\langle\mathcal{O}_{i}\right\rangle \chi^{\prime \prime}(0)
$$

From Eqs. (25) and (31), we arrive at

$$
\chi^{\prime \prime}(0)=\left.\left[\frac{P^{\prime}(z)}{P(z)} \chi^{\prime}(z)\right]\right|_{z \rightarrow 0}=-\frac{4 \sqrt{\alpha}\left\langle\mathcal{O}_{i}\right\rangle \mu_{c}}{\sqrt{1-4 \alpha}-1} \int_{0}^{1} \frac{z^{2 \lambda_{i}-4} P F^{2}}{f} d z
$$

Using the above formula and Eq. (30), we can deduce

$$
\rho=\Gamma(\alpha, m)\left(\mu-\mu_{c}\right)
$$


where $\Gamma(\alpha, m)$ is a function of the Gauss-Bonnet coupling and the scalar field mass

$$
\Gamma(\alpha, m)=\frac{\sqrt{\alpha}}{(\sqrt{1-4 \alpha}-1) \xi(0)} \int_{0}^{1} \frac{z^{2 \lambda_{i}-4} P F^{2}}{f} d z
$$

Fixing $m^{2} L_{\text {eff }}^{2}=-15 / 4$ and $\alpha=0.0001$, for example, we can get $\Gamma(\alpha, m)=1.330$ when $a=0.330$, so that $\rho=1.330\left(\mu-\mu_{c}\right)$ for considering the scalar operator $\left\langle\mathcal{O}_{+}\right\rangle$. This is consistent with the result given in [17]. Here we observed that the Gauss-Bonnet coupling will not alter the result. Our analytic finding of a linear relation between the charge density and the chemical potential $\rho \sim\left(\mu-\mu_{c}\right)$ supports the numerical result reported in [18].

\section{PHASE TRANSITION BETWEEN THE P-WAVE INSULATOR AND SUPERCONDUCTOR}

Since the S-L method is effective to obtain the s-wave holographic insulator and superconductor phase transition, we will use it to investigate analytically the p-wave holographic insulator and superconductor phase transition in the Gauss-Bonnet gravity which has not been constructed as far as we know.

Considering an $S U(2)$ Yang-Mills action in the bulk theory [38]

$$
S=\int d^{5} x \sqrt{-g}\left(-\frac{1}{4} F_{\mu \nu}^{a} F^{a \mu \nu}\right),
$$

where $F_{\mu \nu}^{a}=\partial_{\mu} A_{\nu}^{a}-\partial_{\nu} A_{\mu}^{a}+\epsilon^{a b c} A_{\mu}^{b} A_{\nu}^{c}$ is the $S U(2)$ Yang-Mills field strength and $\epsilon^{a b c}$ is the totally antisym-

metric tensor with $\epsilon^{123}=+1$. The $A_{\mu}^{a}$ are the components of the mixed-valued gauge fields $A=A_{\mu}^{a} \tau^{a} d x^{\mu}$, where $\tau^{a}$ are the three generators of the $S U(2)$ algebra satisfy $\left[\tau^{a}, \tau^{b}\right]=\epsilon^{a b c} \tau^{c}$.

In order to construct a p-wave holographic insulator and superconductor in the Gauss-Bonnet gravity, we adopt the ansatz of the gauge fields as [16, 17, 39-41],

$$
A(r)=\phi(r) \tau^{3} d t+\psi(r) \tau^{1} d x
$$

Here we regard the $U(1)$ symmetry generated by $\tau^{3}$ as the $U(1)$ subgroup of $S U(2)$. The gauge boson with nonzero component $\psi(r)$ along $x$-direction is charged under $A_{t}^{3}=\phi(r)$. According to AdS/CFT correspondence, $\phi(r)$ and $\psi(r)$ are dual to the chemical potential and the $x$-component of some charged vector operator $O$ in the boundary field theory respectively. The condensation of $\psi(r)$ will spontaneously break the $U(1)$ gauge symmetry and lead to a phase transition, which can be interpreted as a p-wave insulator and superconductor phase transition on the boundary.

From the Yang-Mills action (36), we can derive the following equations of motion

$$
\psi^{\prime \prime}+\left(\frac{f^{\prime}}{f}+\frac{1}{r}\right) \psi^{\prime}+\frac{\phi^{2}}{r^{2} f} \psi=0
$$




$$
\phi^{\prime \prime}+\left(\frac{f^{\prime}}{f}+\frac{1}{r}\right) \phi^{\prime}-\frac{\psi^{2}}{r^{2} f} \phi=0
$$

where the prime denotes the derivative with respect to $r$.

In order to solve the above equations of motion, we have to impose the boundary conditions for the $\phi(r)$ and $\psi(r)$ fields at the tip $r=r_{s}$ and at $r \rightarrow \infty$. At the tip $r=r_{s}$, the solutions have the same form just as Eq. (8) for the s-wave holographic insulator and superconductor model. But near the boundary $r \rightarrow \infty$, we have different asymptotic behaviors

$$
\psi=\psi_{0}+\frac{\psi_{2}}{r^{2}}, \quad \phi=\mu-\frac{\rho}{r^{2}}
$$

where $\mu$ and $\rho$ are interpreted as the chemical potential and charge density in the dual field theory, while $\psi_{0}$ and $\psi_{2}$ may be identified as a source and the expectation value of the dual operator, respectively. Since we are interested in the case where the dual operator is not sourced, we will set $\psi_{0}=0$ and have a normalizable solution.

\section{A. Critical chemical potential}

Define the variable $z=r_{s} / r$, the equations of motion (38) and (39) can be expressed in the $\mathrm{z}$ coordinate as

$$
\begin{aligned}
\psi^{\prime \prime}+\left(\frac{f^{\prime}}{f}+\frac{1}{z}\right) \psi^{\prime}+\frac{\phi^{2}}{z^{2} f} \psi & =0, \\
\phi^{\prime \prime}+\left(\frac{f^{\prime}}{f}+\frac{1}{z}\right) \phi^{\prime}-\frac{\psi^{2}}{z^{2} f} \phi & =0,
\end{aligned}
$$

where the prime denotes the derivative with respective to $z$.
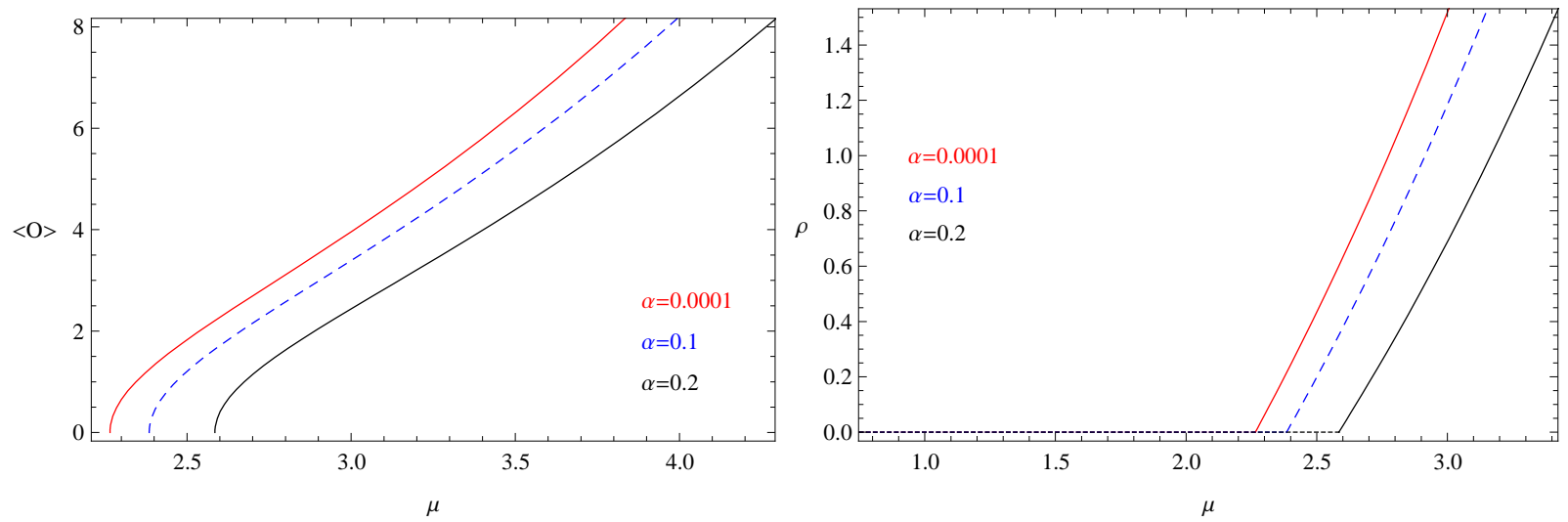

FIG. 1: (color online) The condensates of the operator $\langle\mathcal{O}\rangle=\psi_{2}$ and charge density $\rho$ with respect to the chemical potential $\mu$ for different Gauss-Bonnet couplings $\alpha$ for the p-wave holographic insulator and superconductor model. The three lines from left to right correspond to increasing $\alpha$, i.e., $\alpha=0.0001$ (red), 0.1 (blue and dashed) and 0.2 (black) respectively. 
Similar to the analysis in the previous section, if $\mu \leq \mu_{c}$, the field $\psi$ is nearly zero, i.e., $\psi \simeq 0$. Thus, we can obtain the physical solution $\phi(z)=\mu$ to Eq. (42) when $\mu<\mu_{c}$. This is consistent with the numerical results in Fig. 1 which plot the condensates of the operator $\langle\mathcal{O}\rangle=\psi_{2}$ and charge density $\rho$ with respect to the chemical potential $\mu$ for different Gauss-Bonnet couplings $\alpha$.

As $\mu \rightarrow \mu_{c}$, Eq. (41) will become

$$
\psi^{\prime \prime}+\left(\frac{f^{\prime}}{f}+\frac{1}{z}\right) \psi^{\prime}+\frac{\mu^{2}}{z^{2} f} \psi=0
$$

We can also define a trial function $F(z)$ near the boundary $z=0$ just as in the last section

$$
\psi(z) \sim\langle\mathcal{O}\rangle z^{2} F(z)
$$

with the boundary condition $F(0)=1$ and $F^{\prime}(0)=0$. Therefore, the equation of motion for $F(z)$ is given by

$$
F^{\prime \prime}+\left(\frac{5}{z}+\frac{f^{\prime}}{f}\right) F^{\prime}+\left(\frac{4}{z^{2}}+\frac{2 f^{\prime}}{z f}+\frac{\mu^{2}}{z^{2} f}\right) F=0
$$

Introducing a new function

$$
T(z)=\frac{z^{3}\left(\sqrt{1+4\left(z^{4}-1\right) \alpha}-1\right)}{2 \sqrt{\alpha}}
$$

we can rewrite Eq. (45) as

$$
\left(T F^{\prime}\right)^{\prime}+T\left(\frac{4}{z^{2}}+\frac{2 f^{\prime}}{z f}+\frac{\mu^{2}}{z^{2} f}\right) F=0
$$

Defining the following parameters

$$
U=\frac{4}{z^{2}}+\frac{2 f^{\prime}}{z f}, \quad V=\frac{T}{z^{2} f}
$$

we find that, following the Sturm-Liouville eigenvlaue problem [30], the minimum eigenvalue of $\mu^{2}$ can be obtained from variation of the following functional

$$
\begin{aligned}
\mu^{2}= & \frac{\int_{0}^{1} T\left(F^{\prime 2}-U F^{2}\right) d z}{\int_{0}^{1} V F^{2} d z} \\
= & \frac{1}{4[6+(3 a-8) a] \alpha^{5 / 2}}\left\{4 \sqrt{\alpha}\left[12 \alpha(1-4 a)+a(8-3 a+20 a \alpha)-8 a(1-4 \alpha)^{3 / 2}\right]\right. \\
& \left.+3(1-4 \alpha)\left[4 \alpha+a^{2}(4 \alpha-1)\right] \log \left[\frac{(1-4 \alpha) \alpha}{(2 \alpha+\sqrt{\alpha})^{2}}\right]\right\},
\end{aligned}
$$

where we have assumed the trial function to be $F(z)=1-a z^{2}$ with a constant $a$.

We can easily obtain the minimum eigenvalues of $\mu^{2}$ and the corresponding values of $a$ for different GaussBonnet couplings $\alpha$, for example, $\mu_{\min }^{2}=5.140$ and $a=0.338$ for $\alpha=0.0001, \mu_{\min }^{2}=5.697$ and $a=0.305$ for 
$\alpha=0.1$ and $\mu_{\min }^{2}=6.691$ and $a=0.223$ for $\alpha=0.2$. Thus, we get the critical chemical potential $\mu_{c}=\mu_{\min }$ 17] which has been shown in Table III for fixed value of the Gauss-Bonnet factor $\alpha$. In order to compare with numerical results, we also give the critical chemical potential obtained by using the shooting method. Obviously, the agreement of the analytic results derived from S-L method with the numerical calculation is quite impressive.

TABLE III: The critical chemical potential $\mu_{c}$ obtained by the analytical S-L method (left column) and from numerical calculation (right column) with fixed Gauss-Bonnet coupling for the p-wave holographic insulator and superconductor model. Note that our result reduces to the result in Ref. [16] if $\alpha \rightarrow 0$.

\begin{tabular}{lllllll}
\hline$\alpha$ & \multicolumn{2}{c}{0.0001} & \multicolumn{2}{c}{0.1} & \multicolumn{2}{c}{0.2} \\
\hline$\mu_{c}$ & 2.267 & 2.265 & 2.387 & 2.385 & 2.587 & 2.585 \\
\hline
\end{tabular}

From Table III, we also find that the critical chemical potential increases when the Gauss-Bonnet factor $\alpha$ becomes bigger, which shows that the higher order curvature corrections in general make the condensation harder to form, just as observed for the s-wave holographic insulator and superconductor model. This property agrees well with the numerical result shown in Fig. 1.

\section{B. Critical phenomena}

With Eq. (44), when $\mu \rightarrow \mu_{c}$ the equation of motion (42) can be rewrited as

$$
\phi^{\prime \prime}+\left(\frac{f^{\prime}}{f}+\frac{1}{z}\right) \phi^{\prime}-\frac{\langle\mathcal{O}\rangle^{2} z^{2} F^{2}}{f} \phi=0
$$

Note that the condensation value of $\psi(z)$ is so small, we will expand $\phi(z)$ in small $\langle\mathcal{O}\rangle$ as

$$
\phi(z) \sim \mu_{c}+\langle\mathcal{O}\rangle \chi(z)+\cdots,
$$

with the boundary condition $\chi(1)=0$ at the tip. Using the function defined in Eq. (24), we can get the equation of motion for $\chi(z)$

$$
\left(P \chi^{\prime}\right)^{\prime}-\langle\mathcal{O}\rangle \mu_{c} \frac{z^{2} P F^{2}}{f}=0
$$

Near $z \rightarrow 0$, we can also expand $\phi$ as

$$
\phi(z) \simeq \mu-\rho z^{2} \simeq \mu_{c}+\langle\mathcal{O}\rangle\left[\chi(0)+\chi^{\prime}(0) z+\frac{1}{2} \chi^{\prime \prime}(0) z^{2}+\cdots\right]
$$

Comparing the coefficients of the $z^{0}$ term in both sides of the above formula, we can obtain

$$
\mu-\mu_{c} \simeq\langle\mathcal{O}\rangle \chi(0) .
$$


Considering the following equation for $\xi(z)$

$$
\xi^{\prime \prime}+\left(\frac{f^{\prime}}{f}+\frac{1}{z}\right) \xi^{\prime}-\frac{z^{2} F^{2}}{f}=0
$$

with

$$
\chi(z)=\langle\mathcal{O}\rangle \mu_{c} \xi(z)
$$

we will have

$$
\langle\mathcal{O}\rangle=\frac{1}{\left[\mu_{c} \xi(0)\right]^{1 / 2}}\left(\mu-\mu_{c}\right)^{1 / 2},
$$

where $\xi(0)=c_{1}-\int_{0}^{1}\left[c_{2}+\int_{1}^{z} F(x)^{2} x^{3} d x\right] \frac{d z}{z f(z)}$ with the integration constants $c_{1}$ and $c_{2}$ determined by the boundary condition $\chi(z)$. For example, for the case of $\alpha=0.0001$, we can get $\xi(0)=0.0673$ when $a=0.338$ which results in $\langle\mathcal{O}\rangle \approx 2.560\left(\mu-\mu_{c}\right)^{1 / 2}$. This is in good agreement with the result given in [16, 17].

It should be noted that the relation (57) is valid for all cases considered here, so the condensation $\langle\mathcal{O}\rangle \sim$ $\left(\mu-\mu_{c}\right)^{1 / 2}$ near the critical point for various values of Gauss-Bonnet couplings, which agrees well the numerical results in Fig. 1 that the phase transition between the p-wave holographic insulator and superconductor belongs to the second order and the critical exponent of the system takes the mean-field value $1 / 2$.

From the coefficients of the $z^{1}$ term in Eq. (53), we obtain that $\chi^{\prime}(0) \rightarrow 0$ which is consistent with the following relation by making integration of both sides of Eq. (52)

$$
\left.\left[\frac{\chi^{\prime}(z)}{z}\right]\right|_{z \rightarrow 0}=-\frac{2 \sqrt{\alpha}\langle\mathcal{O}\rangle \mu_{c}}{\sqrt{1-4 \alpha}-1} \int_{0}^{1} \frac{z^{2} P F^{2}}{f} d z
$$

For the coefficients of the $z^{2}$ term in Eq. (53), we have

$$
\rho=-\frac{1}{2}\langle\mathcal{O}\rangle \chi^{\prime \prime}(0)=\Gamma(\alpha)\left(\mu-\mu_{c}\right)
$$

where $\Gamma(\alpha)$ is only the function of the Gauss-Bonnet couplings which can be given by

$$
\Gamma(\alpha)=\frac{\sqrt{\alpha}}{(\sqrt{1-4 \alpha}-1) \xi(0)} \int_{0}^{1} \frac{z^{2} P F^{2}}{f} d z .
$$

For example, we can obtain $\Gamma(\alpha)=1.126$ when $a=0.338$ for $\alpha=0.0001$, i.e., the linear relation $\rho=$ 1.126 $\left(\mu-\mu_{c}\right)$, which agrees well with the result given in [17]. Still we notice that the Gauss-Bonnet coupling will not change the result. The analytic finding of a linear relation between the charge density and the chemical potential $\rho \sim\left(\mu-\mu_{c}\right)$ is consistent with the numerical result presented in Fig. 1 . 


\section{CONCLUSIONS}

We have applied the S-L method to investigate analytically the condensation and critical phenomena of the phase transition between the holographic insulator and superconductor in the Gauss-Bonnet gravity. We found that unlike the analytic matching method, the S-L method is effective to obtain the analytic results in the AdS soliton background both for s-wave (the scalar field) and p-wave (the vector field) models. Different from the AdS black hole in the Gauss-Bonnet gravity, in the AdS soliton spacetime we observed that the S-L method can bring us results of condensation for different values of the scalar field mass satisfying the Breitenlohner-Freedman bound. For the massless scalar field, the information of the Gauss-Bonnet coupling can still be kept in the S-L method. With this analytic method, we also found that it is more appropriate to choose the mass of the scalar field by selecting the value of $m^{2} L_{\text {eff }}^{2}$. The analytic results derived from the S-L method for the s-wave and p-wave holographic insulator/superconductor phase transitions support the numerical computations and show that the higher curvature corrections make it harder for the condensation to form.

Furthermore, comparing with the matching method, we found that in the AdS soliton in the Gauss-Bonnet gravity the S-L method can present us analytic results on critical exponent of condensation operator and the relation between the charge density and the chemical potential near the phase transition point. We observed that effect of the Gauss-Bonnet factor cannot modify the critical phenomena. The analytic results can be

used to back up the numerical findings in both s-wave and p-wave insulator/superconductor models of the Gauss-Bonnet gravity.

\section{Acknowledgments}

This work was supported by the National Natural Science Foundation of China; the National Basic Research of China under Grant No. 2010CB833004, PCSIRT under Grant No. IRT0964, the Construct Program of the National Key Discipline, and Hunan Provincial Natural Science Foundation of China 11JJ7001.

[1] E. Witten, Anti-de Sitter space and holography, Adv. Theor. Math. Phys. 2, 253 (1998).

[2] J. Maldacena, The large-N limit of superconformal field theories and supergravity, Adv. Theor. Math. Phys. 2, 231 (1998) [Int. J. Theor. Phys. 38, 1113 (1999)].

[3] S.S. Gubser, I.R. Klebanov, and A.M. Polyakov, Gauge theory correlators from non-critical string theory, Phys. Lett. B 428, 105 (1998).

[4] S.S. Gubser, Breaking an Abelian gauge symmetry near a black hole horizon, Phys. Rev. D 78, 065034 (2008).

[5] S.A. Hartnoll, C.P. Herzog, and G.T. Horowitz, Building a Holographic Superconductor, Phys. Rev. Lett. 101, 031601 (2008). 
[6] S.A. Hartnoll, C.P. Herzog, and G.T. Horowitz, Holographic Superconductors, J. High Energy Phys. 12, 015 (2008).

[7] S.A. Hartnoll, Lectures on holographic methods for condensed matter physics, Class. Quant. Grav. 26, 224002 (2009).

[8] C.P. Herzog, Lectures on Holographic Superfluidity and Superconductivity, J. Phys. A 42, 343001 (2009).

[9] G.T. Horowitz, Introduction to Holographic Superconductors, arXiv:1002.1722 [hep-th].

[10] T. Nishioka, S. Ryu, and T. Takayanagi, Holographic Superconductor/Insulator Transition at Zero Temperature, J. High Energy Phys. 03, 131 (2010).

[11] S. Surya, K. Schleich, and D.M. Witt, Phase Transitions for Flat Anti-de Sitter Black Holes, Phys. Rev. Lett. 86, 5231 (2001).

[12] G.T. Horowitz and B. Way, Complete Phase Diagrams for a Holographic Superconductor/Insulator System, J. High Energy Phys. 11, 011 (2010).

[13] Y. Peng, Q.Y. Pan, and B. Wang, Various types of phase transitions in the AdS soliton background, Phys. Lett. B 699, 383 (2011); arXiv:1104.2478 [hep-th].

[14] P. Basu, F. Nogueira, M. Rozali, J.B. Stang, and M.V. Raamsdonk, Towards A Holographic Model of Color Superconductivity, New J. Phys. 13, 055001 (2011); arXiv:1101.4042 [hep-th].

[15] Y. Brihaye and B. Hartmann, Holographic superfluid/fluid/insulator phase transitions in $2+1$ dimensions, Phys. Rev. D 83, 126008 (2011); arXiv:1101.5708 [hep-th].

[16] A. Akhavan and M. Alishahiha, P-Wave Holographic Insulator/Superconductor Phase Transition, Phys. Rev. D 83, 086003 (2011); arXiv:1011.6158 [hep-th].

[17] R.G. Cai, H.F. Li, and H.Q. Zhang, Analytical Studies on Holographic Insulator/Superconductor Phase Transitions, Phys. Rev. D 83, 126007 (2011); arXiv:1103.5568 [hep-th].

[18] Q.Y. Pan, B. Wang, E. Papantonopoulos, J. Oliveria, and A.B. Pavan, Holographic Superconductors with various condensates in Einstein-Gauss-Bonnet gravity, Phys. Rev. D 81, 106007 (2010).

[19] R. Gregory, S. Kanno, and J. Soda, Holographic Superconductors with Higher Curvature Corrections, J. High Energy Phys. 10, 010 (2009).

[20] S. Kanno, A Note on Gauss-Bonnet Holographic Superconductors, Class. Quant. Grav. 28, 127001 (2011); arXiv:1103.5022 [hep-th].

[21] X.H. Ge, B. Wang, S.F. Wu, and G.H. Yang, Analytical study on holographic superconductors in external magnetic field, J. High Energy Phys. 08, 108 (2010); arXiv:1002.4901 [hep-th].

[22] X.H. Ge, Analytical calculation on critical magnetic field in holographic superconductors with backreaction, arXiv:1105.4333 [hep-th].

[23] G. Siopsis and J. Therrien, Analytic calculation of properties of holographic superconductors, J. High Energy Phys. 05, 013 (2010).

[24] G. Siopsis, J. Therrien, and S. Musiri, Holographic superconductors near the Breitenlohner-Freedman bound, arXiv:1011.2938 [hep-th].

[25] H.B. Zeng, X. Gao, Y. Jiang, and H.S. Zong, Analytical Computation of Critical Exponents in Several Holographic Superconductors, J. High Energy Phys. 05, 002 (2011); arXiv:1012.5564 [hep-th].

[26] H.F. Li, R.G. Cai, and H.Q. Zhang, Analytical Studies on Holographic Superconductors in Gauss-Bonnet Gravity, J. High Energy Phys. 04, 028 (2011); arXiv:1103.2833 [hep-th].

[27] Y.Q. Liu, Q.Y. Pan, B. Wang, and R.G. Cai, Dynamical perturbations and critical phenomena in Gauss-BonnetAdS black holes, Phys. Lett. B 693, 343 (2010).

[28] R.G. Cai, S.P. Kim, and B. Wang, Ricci flat black holes and Hawking-Page phase transition in Gauss-Bonnet gravity and dilaton gravity, Phys. Rev. D 76, 024011 (2007).

[29] R.G. Cai, Gauss-Bonnet black holes in AdS spaces, Phys. Rev. D 65, 084014 (2002).

[30] I.M. Gelfand and S.V. Fomin, Calculaus of Variations, Revised English Edition, Translated and Edited by R.A. Silverman, Prentice-Hall, Inc. Englewood Cliff, New Jersey (1963).

[31] Y. Brihaye and B. Hartmann, Holographic Superconductors in $3+1$ dimensions away from the probe limit, Phys. Rev. D 81, 126008 (2010); arXiv:1003.5130 [hep-th].

[32] L. Barclay, R. Gregory, S. Kanno, and P. Sutcliffe, Gauss-Bonnet Holographic Superconductors, J. High Energy Phys. 12, 029 (2010); arXiv:1009.1991 [hep-th].

[33] Q.Y. Pan and B. Wang, General holographic superconductor models with Gauss-Bonnet corrections, Phys. Lett. B 693, 159 (2010).

[34] R.G. Cai, Z.Y. Nie, and H.Q. Zhang, Holographic p-wave superconductors from Gauss-Bonnet gravity, Phys. Rev. D 82, 066007 (2010).

[35] M. Siani, Holographic Superconductors and Higher Curvature Corrections, J. High Energy Phys. 12, 035 (2010); arXiv:1010.0700 [hep-th].

[36] D. Momeni, M.R. Setare, and N. Majd, Holographic superconductors in a model of non-relativistic gravity, J. High Energy Phys. 05, 118 (2011).

[37] P. Breitenloher and D.Z. Freedman, Stability in gauged extended supergravity, Ann. Phys. 144, 249 (1982).

[38] S.S. Gubser, Colorful Horizons with Charge in Anti-de Sitter Space, Phys. Rev. Lett. 101, 191601 (2008).

[39] S.S. Gubser and S.S. Pufu, The gravity dual of a p-wave superconductor, J. High Energy Phys. 11, 033 (2008).

[40] P. Basu, J. He, A. Mukherjee, and H.H. Shieh, Hard-gapped Holographic Superconductors, Phys. Lett. B 689, 45 (2010).

[41] M. Ammon, J. Erdmenger, V. Grass, P. Kerner, and A. O'Bannon, On Holographic p-wave Superfluids with 
Back-reaction, Phys. Lett. B 686, 192 (2010). 